\begin{tabular}{lll}
\hline Kai-Håkon Carlsen & $\begin{array}{l}\text { Oslo University Hospital, } \\
\text { Rikshospitalet, Department } \\
\text { of Paediatrics, P.OB Box 450 } \\
\text { Nydalen, 0424 Oslo, Norway. }\end{array}$ & k.h.carlsen@medisin.uio.no \\
&
\end{tabular}

\title{
GPs Meet Rare Lung Disorders Task Force factsheet: lung disease in children with immunodeficiences
}

Chronic lung diseases in children are classified as primary and secondary lung disease. Secondary lung disease may develop due to several systemic disorders including primary ciliary dyskinesia, cystic fibrosis, due to oesophagus atresia and tracheaoesophagal fistula, neuromuscular disease and immunodeficiencies. In the lung, there are several different defence mechanisms against infections, both local and general. In this respect we relates to systemic causes of reduced immune defence.

\section{Primary immunodeficiences}

Primary immunodeficiencies may present with different clinical pictures due to different pathogenic mechanisms involved. There are disorders of antibody function, T-cell disorders, phagocytic disorders and others including severe combined immunodeficiency (SCID).

\section{Antibody function disorders}

The antibody function disorders are the most common and include X-linked and non-X-linked a- and hypo- $\gamma$-globulinaemia, common variable hypo- $\gamma$-globulinaemia, immunoglobulin (Ig)G subclass deficiencies, IgA deficiencies, hyperIgM syndrome and hyper-IgE syndrome.

\section{Main signs}

Although variable, the main signs of systemic immunodeficiency are increased number of infections involving several different organs including both the upper and lower respiratory tract. Chronic otitis media and rhinosinusitis are characteristic signs. Several of the immunodeficiencies present with recurrent lower respiratory tract infections (LRTI). Repeated LRTIs during childhood should always alert the physician to the possibility of immunodeficiency.

\section{Diagnosis}

The awareness of immunodeficiencies is the most important basis for the diagnosis. Early treatment with immunosubstitution is very important to prevent the development of pulmonary sequelae.

In addition to the primary diagnosis of the immunodeficiency itself, it is important to assess the degree of pulmonary involvement. This includes clinical examination as well as assessment of pulmonary function and radiological examination including highresolution computed tomography scans.

Exercise testing may be performed to assess fitness and exercise limitation due to reduced baseline lung function with flow limitation during exercise or exercise-induced bronchoconstriction. 


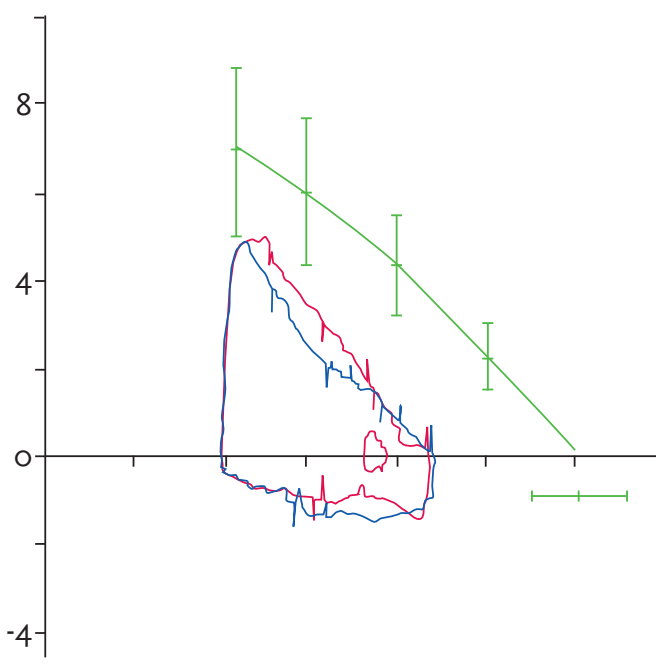

Figure 1

Lung function before and after inhaled salbutamol in a 16-year-old male with hypo- $\gamma$-globulinaemia and immunoglobulin $G$ subclass deficiency. The spirometry demonstrates a restrictive lung disorder with reduced vital capacity and without signs of obstructive airways disease. No reversibility to salbutamol.

\section{Clinical respiratory symptoms}

Clinical respiratory symptoms will depend upon type of immunodeficiency and number and severity of the respiratory infections. Repeated pneumonias will lead to increasing damage to the lungs. Increased mucous secretions will also appear during good periods without infections with audible rhonchi and sibilant, low-pitched rhonchi. Parents frequently are disturbed in their sleep and lie awake during nights listening to the respiratory sounds of their child. A frequent complication is recurrent atelectasis and the gradual development of bronchiectasis. If the damage to the respiratory tract and the lungs has progressed, the lung disease may be self-perpetuating even after treatment with immunosubstitution has been started.

Lung function measurements may demonstrate signs of both obstructive and restrictive ventilatory impairment (figure 1).

In such cases treatment of the primary disease, the immunodeficiency, should be started, as well as treatment of the respiratory symptoms and signs. Treatment is dependent upon clinical symptoms and findings of lung function and radiographic examination. Both bronchodilator and anti-inflammatory treatment with inhaled steroids may be indicated. Mobilisation of respiratory secretions should be given by lung physiotherapy after bronchodilation. In the case of atelectasis, inhaled antibiotic therapy as in cystic fibrosis should be considered. Inhaled tobramycin is a good choice. This may markedly improve symptoms, reduce bronchial secretions and reduce bronchiectasis as demonstrated by high-resolution chest computed tomography.

An active life with frequent physical activity should be recommended for an adolescent with immunodeficiency, both to mobilise bronchial secretions and to improve fitness and quality of life.

Most importantly, the caring physician should be aware of the possibility of immunodeficiency and investigate in order to make an early diagnosis, thus improving the prognosis for a child with immunodeficiency.

\section{Further reading}

1. Balbi B et al. Immunology and defence mechanisms. In ERS Handbook in Respiratory Medicine 2nd edition. Palange $\mathrm{P}$ and Simonds A, eds. 39--44. ISBN 978-1-84984-023-1.

2. Stafler P, Carr SB. Non-cystic fibrosis bronchiectasis: its diagnosis and management. Arch Dis Child Educ Pract Ed 2010; 95: 73-82.

3. Gregersen $\mathrm{S}$, Holm AM, Fevang B, et al. Lung disease, T-cells and inflammation in common variable immunodeficiency disorders. Scand J Clin Lab Invest 2013; 73: 514-522.

4. Roxo P Jr, Torres LA, Menezes UP, et al. Lung function in hyper IgE syndrome. Pediatr Pulmonol 2013; 48: 81-84.

5. Kim HY, Kwon JW, Seo J, et al. Bronchiectasis in children: 10-year experience at a single institution. Allergy Asthma Immunol Res 2011; 3: 39-45.

6. Yong PL, Orange JS, Sullivan KE. Pediatric common variable immunodeficiency: immunologic and phenotypic associations with switched memory B cells. Pediatr Allergy Immunol 2010; 21: 852-858.

7. Haidopoulou K, Calder A, Jones A, et al. Bronchiectasis secondary to primary immunodeficiency in children: longitudinal changes in structure and function. Pediatr Pulmonol 2009; 44: 669-675.

8. Freeman, A. F. and S. M. Holland (2008). "The hyper-IgE syndromes." Immunol Allergy Clin North Am 28(2): 277291, viii. 